\title{
Threshold Effect on Particle Tracking Algorithms
}

\author{
Harun Yücel ${ }^{1, a)}$ and Sabareesh K. P. Velu ${ }^{2,3, b)}$ \\ ${ }^{1}$ Vocational School of Health Services, Bayburt University, 69000-Bayburt, Turkey \\ ${ }^{2}$ Department of Physics, Bilkent University, 06800-Ankara, Turkey \\ ${ }^{3}$ University of Information Science and Technology "St. Paul the Apostle", 6000-Ohrid, Macedonia

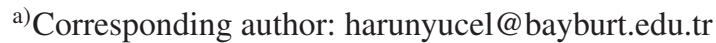 \\ b) sabareesh.velu@uist.edu.mk
}

\begin{abstract}
Automated particle tracking algorithms are widely used by soft matter physicists as a research tool to detect and construct the trajectories of micron-sized particles in fluids. Analyzing these trajectories will uncover the physics of the investigated particles mainly on the type of motion they undergo making them suitable for potential applications. A plethora of methods has been proposed and used for detection and tracking. In this work, we examine the performance of two commonly used tracking algorithms in terms of threshold dependencies in digital video images. One of them is the centroid method (CM), a well-known and used algorithm and the other is radial symmetry method (RSM) which is recently proposed. Here, we generate the synthetic digital video images consisting of randomly placed multiple particles and compare the absolute errors on the particle detection by varying threshold values. Our results suggest that both algorithms show dependence on the threshold value and on comparison RSM algorithm performs better than the CM algorithm when the noise level is zero. Moreover, the measured absolute errors show a strong dependence on threshold values when noise levels are increased (up to 20) especially for the RSM algorithm.
\end{abstract}

\section{INTRODUCTION}

Particle detection and tracking is an important tool to obtain quantitative information about soft matter systems [1, 2, 3] observed by DVM (Digital Video Microscopy). In DVM, micron-sized particles suspended in a liquid are imaged over time by a microscope system that includes microscope objective and digital camera. The resulted time-lapse images are then analyzed using particle detection algorithms to trace $2 \mathrm{D} / 3 \mathrm{D}$ particle positions and trajectories. The recovered trajectories include all information about the interaction between particles or about the interaction between the particles and surrounding liquid or about an external force acting on the particles like optical, electrical forces $[4,5]$. Therefore, the detection and tracking algorithms and their accuracy on the particle position is essential to understand physics underlying with the micro-world making them suitable for healthcare, sustainable and security applications [6].

When looking at the literature, it is possible to find many tracking algorithms that use different approaches and are adapted different experimental conditions. For the studies on colloids in which the particles are generally spherical in shape, there are several commonly used basic algorithms such as the centroid algorithm $(\mathrm{CM})$, the radial symmetry centers algorithm (RSM) and the Gaussian fitting algorithm. These algorithms are comprehensively reviewed in [7, $8]$. Here, we focus only two widely used algorithms, CM as explained in $[9,10]$ and RSM as reported in $[11,9$, 12], because these algorithms are suitable with threshold application which is a pre-processing technique in image segmentation [13]. Threshold technique presents an easy and useful way to identify spherical particles, especially for multiple particles condition.

In DVM, the intensity profile of a single spherical microparticle in the image could be presented as Gaussian Bessel function [14], approximately. The central region of the particle will have a bright point with respect to background intensity (usually black or gray). On applying a threshold value to the image, it eliminates the background intensity (black or gray region) and accounts only the bright region of the particles which is called as the "active area of the particle". The center of the active area (based on intensity) will be treated as particle position that can be obtained by both CM and RSM algorithms. For the case of multiple particles condition, both methods work with a labelling algorithm. However, using a threshold value in detection algorithms may cause an error on the accuracy

Turkish Physical Society 34th International Physics Congress (TPS34) AIP Conf. Proc. 2042, 020004-1-020004-4; https://doi.org/10.1063/1.5078876 Published by AIP Publishing. 978-0-7354-1768-7/\$30.00 

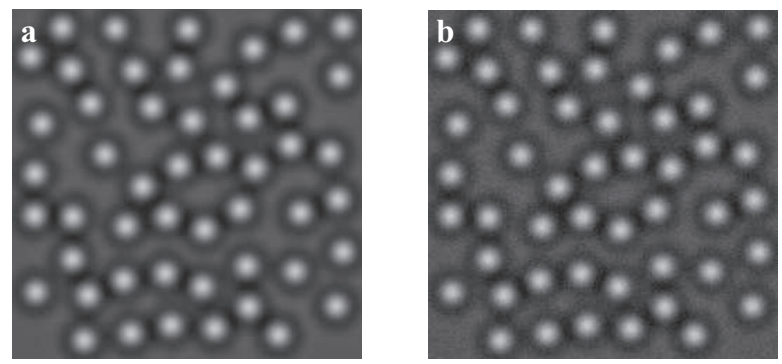

FIGURE 1. Simulated images consisting multiple particles with (a) noise level $=0$ and (b) noise level $=20$.

of the particle position. Therefore, while choosing a threshold value, it is important to have the knowledge about its effect on the accuracy of the particle position. For CM, the accuracy on threshold effect is predictable as higher the threshold values will decrease the size of the active area. For the case of RSM, it is difficult to predict about the threshold effect due to the fact that the application of RSM with a threshold was performed first in [15, 16] and it never be tested before. This makes the motivation of this work to investigate the threshold effect on the accuracy of the particle position using RSM and comparing the results with CM.

With this objective, we generated simulated images that consisting multiple particles based on the intensity profile curve extracted from the experimentally recorded single particle image using DVM. Then, we determine the absolute errors of CM and RSM algorithm by varying threshold values and image noise levels. The obtained results on both algorithms show clearly the accuracy of particle position depends on the threshold value. However, it should be noted that the accuracy error from RSM is much less compared to CM when image noise level is zero. Moreover, we also observed the dependency of RSM on threshold value when image noise level increased up to 20.

\section{MATERIALS AND METHODS}

For generating the simulated image, we used the intensity profile of a single particle that is recorded experimentally using DVM. The experimental images were obtained under white light (incoherent) illumination with 100X (NA = 1.3) oil immersion microscope objective coupled with CCD camera using a diluted aqueous solution containing 2.06 $\mu m$ sized silica particles purchased from Microparticles GMBH. The experimentally imaged particle's intensity profile is then fitted with Gaussian-Bessel function and with the obtained parameters, we generated the simulated particle for this work. The formulation of image generation with multiple particles can be given as $I(x, y)=\sum_{i=1}^{N} I_{\exp }\left(x_{i}, y_{i}\right)+I_{0}+$ Noise, where $I_{\text {exp }}\left(x_{i}, y_{i}\right)$ denotes the experimentally obtained particle intensity localized at $\left(x_{i}, y_{i}\right) . I_{0}$ is the background intensity. $x$ and $y$ correspond to column and row index of the image array, respectively. For adding noise in the image, we used random fluctuations whose amplitude (noise level) is in gray scale.

First, we considered $250 \times 250$ size image where we placed randomly 50 particle intensity as shown in Figure 1. Figure 1a shows the original simulated image without noise and Figure $1 \mathrm{~b}$ shows the same image with noise level 20. In addition, we also simulated the images with noise level 5, 10 (not shown in Figure 1) to investigate them systematically.

For calculating the particle centers, we consider the image $I(i, j)$ which $i=1,2,3, \ldots, N$ and $j=1,2,3, \ldots, M$ are row and column indexes of the images. For $t h$ threshold parameter, we follow the equation as

$$
L(i, j)= \begin{cases}1 & I(i, j) \geq t h \\ 0 & I(i, j)<t h\end{cases}
$$

where $L$ denotes the active areas of the particles. After applying the threshold, the pixel connectivity algorithm labels each region of particles like $k=1,2,3, \ldots$. Therefore, we can extract binary images of each particle to obtain the active area arrays $L_{k}$ which have the same size as the image. For CM algorithm we calculate the center $\left(x_{k}^{c}, y_{k}^{c}\right)$ for $k$ th particle by means of the following equation

$$
x_{k}^{c}=\frac{\sum_{i, j} j L_{k}(i, j) I(i, j)}{\sum_{i, j} j} \quad, \quad y_{k}^{c}=\frac{\sum_{i, j} i L_{k}(i, j) I(i, j)}{\sum_{i, j} i}
$$



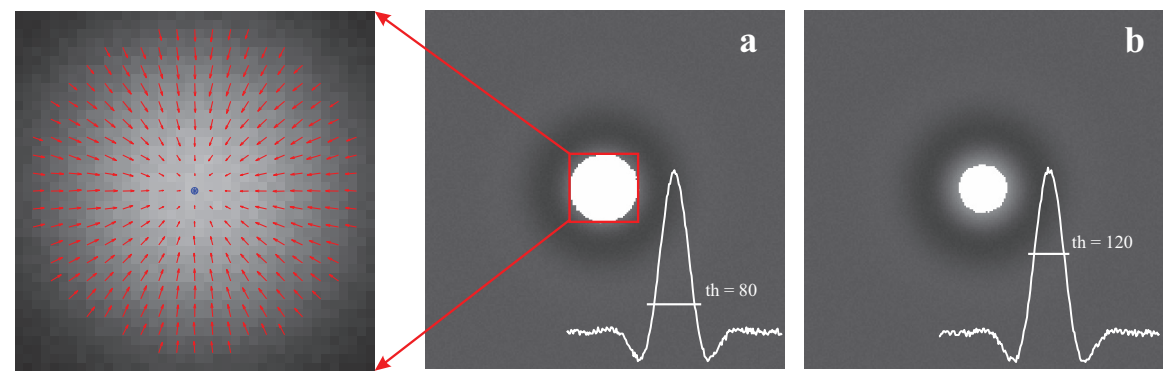

FIGURE 2. Graphical representation of RSM algorithm showing particle intensity gradient vectors (in red arrows) and particle center (as a blue dot) by using the intensity information within the active area (white region). Particle's intensity profile showing (a) at threshold value $t h=80$, (b) at threshold value $t h=120$ where the active are of the particle is smaller than that of $t h=80$.

For RSM algorithm, we calculate image gradient vector elements by using [12]

$$
g_{x}=\left[\begin{array}{ccccc}
1 & 1 & 0 & -1 & -1 \\
1 & 1 & 0 & -1 & -1 \\
1 & 1 & 0 & -1 & -1
\end{array}\right] * I \quad, \quad g_{y}=\left[\begin{array}{ccc}
1 & 1 & 1 \\
1 & 1 & 1 \\
0 & 0 & 0 \\
-1 & -1 & -1 \\
-1 & -1 & -1
\end{array}\right] * I
$$

operators and we determine the slopes $(m(i, j))$ of the gradient vectors and weight function $\left(w_{k}(i, j)\right)$ for $k$ th particle as presented by

$$
m(i, j)=g_{y}(i, j) / g_{x}(i, j) \quad, \quad w_{k}(i, j)=L_{k}(i, j) \sqrt{g_{x}(i, j)^{2}+g_{y}(i, j)^{2}}
$$

then, the analytic solution of RSM algorithm is obtained by the equations [9, 12]

$$
A_{k}=\sum_{i, j}^{N, M} \frac{w_{k}(i, j) m(i, j)^{2}}{m(i, j)^{2}+1}, \quad B_{k}=-\sum_{i, j}^{N, M} \frac{w_{k}(i, j) m(i, j)}{m(i, j)^{2}+1}, \quad C_{k}=-\sum_{i, j}^{N, M} \frac{w_{k}(i, j) m(i, j)}{m(i, j)^{2}+1}, \quad D_{k}=\sum_{i, j}^{N, M} \frac{w_{k}(i, j)}{m(i, j)^{2}+1}
$$

and

$$
E_{k}=\sum_{i, j}^{N, M} \frac{w_{k}(i, j) m(i, j)(m(i, j) j-i)}{m(i, j)^{2}+1}, \quad F_{k}=-\sum_{i, j}^{N, M} \frac{w_{k}(i, j)(m(i, j) j-i)}{m(i, j)^{2}+1}
$$

and the solution can be written as

$$
\left[\begin{array}{ll}
A_{k} & B_{k} \\
C_{k} & D_{k}
\end{array}\right]\left[\begin{array}{l}
x_{k}^{c} \\
y_{k}^{c}
\end{array}\right]=\left[\begin{array}{c}
E_{k} \\
F_{k}
\end{array}\right] \Rightarrow\left[\begin{array}{c}
x_{k}^{c} \\
y_{k}^{c}
\end{array}\right]=\frac{1}{A_{k} D_{k}-B_{k} C_{k}}\left[\begin{array}{cc}
D_{k} & -B_{k} \\
-C_{k} & A_{k}
\end{array}\right]\left[\begin{array}{c}
E_{k} \\
F_{k}
\end{array}\right]
$$

A graphical representation of RSM algorithm is shown in Figure 2 shows the intensity distribution of the particle in the active area (gradient vectors in red arrows) and the particle center (blue dot) appearing as the intersection point of the vectors. In Figure $2 \mathrm{a}$, one can notice the active area, i.e., a white region in the particle image after applying threshold value $t h=80$. In Figure 2b, it can be noticed that the size of the active area becomes smaller for the threshold value $t h=120$, which may cause error in the calculation of the center coordinates.

\section{RESULTS AND DISCUSSIONS}

The generated simulated image of randomly placed multiple particles as mentioned in materials and methods is then used to calculate the particle positions using CM and RSM algorithms independently. Determining the particle positions, we evaluate the absolute errors on each particle and obtain the average absolute errors over all the particles. The results of obtained average absolute errors are plotted versus the applied threshold th value as shown in Figure 3. Figure 3 a corresponds for the simulated image with zero noise level. 

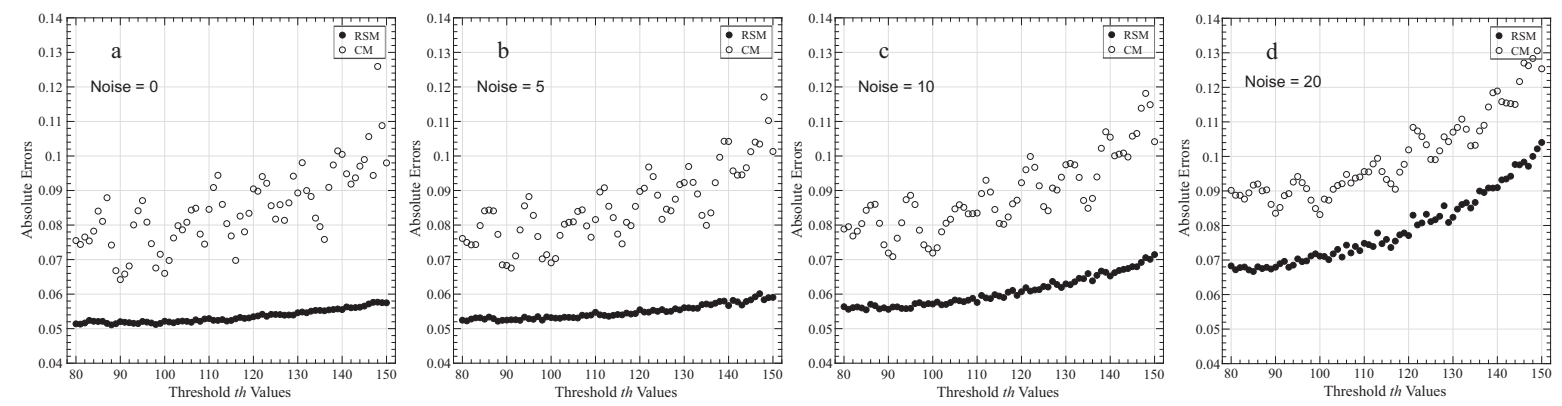

FIGURE 3. Plot of average absolute errors obtained for CM and RSM algorithms by varying the threshold values between 80 to 150 with noise level $=0(\mathrm{a}),=5(\mathrm{~b}),=10(\mathrm{c})$, and $=20$ (d) respectively.

For this case, $\mathrm{CM}$ algorithm shows a high dependence on the threshold values while the RSM algorithm shows less dependence in which the error difference is only with in 0.01 for all the threshold values. Similar analysis is repeated for the simulated images with noise level $=5$ (Figure 3b), $=10$ (Figure 3c), and $=20$ (Figure 3d) respectively. The results reveal strong dependence on the increase in threshold when noise level is increased especially for RSM when compared to CM algorithm.

\section{CONCLUSIONS}

In this work, we present the threshold effect on the particle tracking algorithms such as CM and RSM. Our results conclude that both CM and RSM show the threshold dependence in terms of absolute error (i.e., accuracy on the particle position) first for zero image noise level. However, on comparison, RSM performs better (less error) than CM algorithm as reported in [9]. Our results suggest to choose the threshold value as minimum to minimize the error on both CM and RSM particle detection algorithms. Secondly, adding image noise (up to 20) also effects the absolute errors especially for RSM when compared to CM algorithm. The possible reason in RSM algorithm could be caused due to the gradient vector calculation (see Eq. 3). Therefore it is mandatory to use noise reduction methods within the gradient vector calculation that could provide better accurate results for the case of RSM algorithm.

\section{REFERENCES}

[1] S. K. P. Velu, arXiv:1801.04135 (2018).

[2] T. Andac, P. Weigmann, S. K. P. Velu, E. Pince, A. Callegari, G. Volpe, and G. Volpe, arXiv:1803.02619 (2018).

[3] S. K. P. Velu, M. Yan, K.-P. Tseng, K.-T. Wong, D. M. Bassani, and P. Terech, Macromolecules 46, 1591$1598(2013)$.

[4] E. Pinçe, S. K. P. Velu, A. Callegari, P. Elahi, S. Gigan, G. Volpe, and G. Volpe, Nature Communications 7, p. 10907 (2016).

[5] S. Nöjd, P. S. Mohanty, P. Bagheri, A. Yethiraj, and P. Schurtenberger, Soft Matter 9, 9199-9207 (2013).

[6] C. Bechinger, R. Di Leonardo, H. Löwen, C. Reichhardt, G. Volpe, and G. Volpe, Reviews of Modern Physics 88, p. 045006 (2016).

[7] C. Manzo and M. F. Garcia-Parajo, Reports on Progress in Physics 78, p. 124601 (2015).

[8] H. Shen, L. J. Tauzin, R. Baiyasi, W. Wang, N. Moringo, B. Shuang, and C. F. Landes, Chemical Reviews 117, 7331-7376 (2017).

[9] R. Parthasarathy, Nature Methods 9, 724-726 (2012).

[10] J. Fish and J. Scrimgeour, Applied Optics 54, p. 6360 (2015).

[11] T. Peng, A. Balijepalli, S. K. Gupta, and T. LeBrun, Journal of Computing and Information Science in Engineering 7, 330-338 (2007).

[12] H. Ma, F. Long, S. Zeng, and Z.-L. Huang, Optics letters 37, 2481-2483 (2012).

[13] K. Bhargavi and S. Jyothi, International Journal of Innovative Research and Development 3 (2014).

[14] J. Baumgartl and C. Bechinger, Europhysics Letters (EPL) 71, 487-493 (2005).

[15] H. Yücel and N. T. Okumuşoğlu, Applied Optics 55, p. 7861 (2016).

[16] H. Yücel and N. T. Okumuşoğlu, Journal of Physics: Condensed Matter 29, p. 465101 (2017). 